\title{
Planung der Spitalentlassung bei schwerer, unheillbarer Krankheit
}

\author{
Ziel des vorgestellten Projektes war, das Austrittsmanagement für unheilbar Kranke \\ so anzupassen, dass ihre Wünsche zur Gestaltung der letzten Lebensphase und zum \\ Sterbeort erfüllt werden können. Ein konkretes Austrittsprozedere wurde erarbeitet \\ und erfolgreich umgesetzt.
}

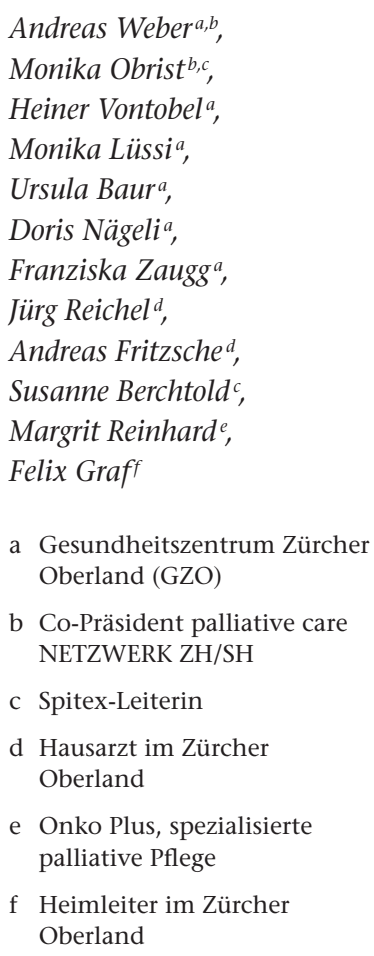

Interessenbindungen: keine

Korrespondenz: Dr. med. Andreas Weber FMH Anästhesie Stationsstrasse 77 8623 Wetzikon Tel. 0844001144 Fax 0442742559 andreas.weber@medix.ch
Die wichtigsten Betreuungsziele von schwer und unheilbar Kranken, nämlich nicht zu leiden und die verbleibende Lebenszeit wenn möglich zu Hause zu verbringen [1], werden bei weniger als $40 \%$ der Patienten erreicht. Über 50\% der Menschen sterben im Kanton Zürich im Spital. Zwischen 40 und 80\% der Patienten mit fortgeschrittenen Tumorerkrankunken leiden unter signifikanten Schmerzen und weiteren Symptomen [2]. Gründe der fehlenden Zielerreichung am Lebensende sind ungenügende Symptomkontrolle zu Hause, mangelnde Aufklärung über den Verlauf der Krankheit und über Möglichkeiten, aber auch Grenzen, diesen Verlauf zu beeinflussen. Bei akuter Verschlechterung des Zustandes ist ein Grund oft die fehlende Erreichbarkeit eines über die Ziele und Probleme des Patienten informierten Arztes und Überforderung der Angehörigen.

Die meisten Patienten werden in ihrem letzten Lebensjahr mindestens einmal, meist mehrmals hospitalisiert [3]. Der Spitalaufenthalt könnte ein geeigneter Moment sein, um persönliche Werte und Ziele in Anbetracht der Prognose zu klären und Chancen und Risiken möglicher Behandlungsoptionen abzuwägen. Palliative Patienten verlassen das Spital nicht gesund, aufgrund der unaufhaltbaren Progression der Krankheit und dem Wunsch, die letzte Lebenszeit zu Hause zu verbringen, manchmal sogar kränker als beim Spitaleintritt. Mehr noch als bei anderen Patientengruppen ist es wichtig, dass der Austritt mit den nachversorgenden Fachleuten gut abgesprochen und sorgfältig geplant wird, damit auch bei weiterer Verschlechterung des Gesundheitszustandes eine Betreuung zu Hause oder im Pflegeheim möglich bleibt.

Ziel des vorliegenden Projektes war, in einem Akutspital das Austrittsmanagement für unheilbar Kranke mit begrenzter Lebenserwartung so anzupassen, dass die Wünsche dieser Patientinnen und Patienten bezüglich Gestaltung der letzten Lebensphase und Ort des Sterbens erreicht werden. Zudem sollte die Zusammenarbeit zwischen ambulant und stationär tätigen Leistungserbringern optimiert werden.

\section{Zusammenfassung}

Viele Menschen leiden in der letzten Lebensphase unter Schmerzen und anderen Beschwerden und die meisten sterben nicht dort, wo sie es sich wünschen. Im vorliegenden Projekt wurde untersucht, ob bei hospitalisierten Patientinnen und Patienten mit einer geschätzten Lebenserwartung von unter einem Jahr eine sorgfältige Planung der Entlassung mit Massnahmenplan für mögliche Komplikationen und Organisation eines 24 Stunden verfügbaren Betreuungsteams (Hausarzt, Spitex und Palliative Care-Spezialisten) zu einem besseren Outcome führen.

Während der einjährigen Pilotphase wurden $\mathbf{4 0}$ meist krebskranke Patienten für eine entsprechende Entlassungsplanung vorgeschlagen. Alle befanden sich bereits in einem sehr fortgeschrittenen Stadium ihrer Krankheit und lebten durchschnittlich nur noch 30 Tage. Bei einem Drittel der Patienten war eine Entlassung aus dem Spital nicht mehr möglich. Bei den anderen zwei Dritteln war eine Spitalentlassung, meistens nach Hause, möglich. $\mathbf{8 0} \%$ der nach Hause entlassenen Patienten wollten auch zu Hause sterben. Bis auf eine Ausnahme konnte dieser Wunsch erfüllt werden. Die Zusammenarbeit zwischen ambulant und stationär tätigen Fachleuten konnte wesentlich verbessert werden.

\section{Patienten, Material, Methode}

Für die Durchführung dieses Projektes wurde das Gesundheitszentrum Zürcher Oberland, ein Regionalspital mit einem Einzugsgebiet von ca. 100000 Perso- 
nen auf $180 \mathrm{~km}^{2}$, ca. 80 Hausarztpraxen, 11 Spitexorganisationen und 15 Alters- und Pflegeheimen gewählt. Das Projekt wurde in zwei Phasen durchgeführt. In der ersten Phase von Januar bis November 2008 wurde eine Arbeitsgruppe, bestehend aus Ärzten und Pflegenden des Spitals, Sozialdienst, Spitexlei- festgehalten. Explizit wird gefragt, wo der Patient seine letzte Lebenszeit verbringen und wo er sterben möchte.

- Bei Austritt ins Pflegeheim oder sonst schwierigen, sozialen Situationen wird die Patientenberatung beigezogen.

\section{Eine gute Planung der Spitalentlassung ermöglicht in den meisten Fäl- len, dass Patienten ihr Leben am gewünschten Ort beenden können}

terinnen, Hausärzten, Heimleitern zusammengestellt. Diese Arbeitsgruppe untersuchte retrospektiv verschiedene Fallbeispiele, bei denen die Wünsche der Patienten in der letzten Lebensphase offensichtlich nicht erfüllt werden konnten, und diskutierte Massnahmen, die zu einem besseren Resultat hätten führen können. Basierend auf diesen Analysen und auf Vorgaben aus anderen Schnittstellenkonzepten [4] wurde ein SollProzess für das Austrittsmanagement dieser Patienten erstellt:

- Am Morgenrapport der Medizin, wenn die neu eingetretenen Patienten vorgestellt werden, wird der zuständige Kaderarzt bei Patienten mit metastasierendem Karzinom oder 3. Hospitalisation wegen COPD oder Herzinsuffizienz gefragt: «Ist das ein Patient, bei dem es dich nicht erstaunen würde, wenn er innerhalb eines Jahres stirbt?»

- Wird die Frage mit ja beantwortet, führt der Palliative care Konsiliararzt (PCKA) ein Gespräch mit dem Patienten und seinen Angehörigen. Es werden Fragen zum weiteren Verlauf, zu Chancen und Risiken möglicher Interventionen zur Beeinflussung der Krankheit und deren Komplikationen besprochen. Die Bedürfnisse, Wertvorstellungen und Ziele des Patienten werden erfasst und schriftlich

\section{Abb. 1}

Patientenweg: Anzahl ins Pilotprojekt eingeschlossene und dann entlassene, bzw. nicht mehr entlassene Patienten, mit gewünschtem und erfolgtem Sterbeort.

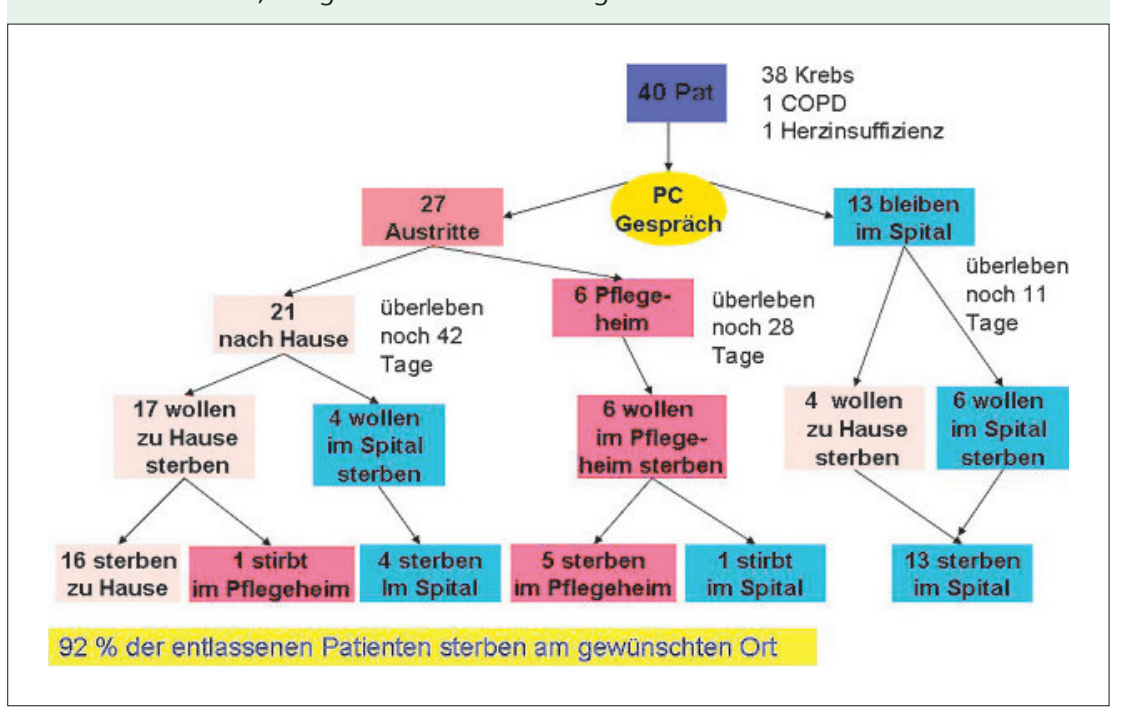

- Vier Tage vor Entlassung werden Hausarzt und Spitex resp. Pflegeheim telefonisch kontaktiert, zwecks Sicherstellung eines 24-Stunden-Dienstes durch informierte und kompetente Pflegende und Ärzte. Den Fachleuten steht auch nach Spitalentlassung der PCKA sowie bei Bedarf die Onko Plus (in Palliative care spezialisierte Pflegefachleute) im Sinne eines mobilen Palliative care-Teams zur Abdeckung des 24-Stunden-Dienstes und bei schwieriger Symptomkontrolle zur Seite.

- Möglicherweise eintretende Notfallsituationen werden mit Patient, Angehörigen und Fachleuten vorbesprochen und entsprechende Massnahmen geplant und wo nötig vorbereitet. Unterstützung der Angehörigen wird für verschiedene Verlaufsszenarien geplant. Die Rolle der verschiedenen Beteiligten wird geklärt und der rasche Informationsfluss in der Regel per E-Mail unter allen vereinbart und im Betreuungsplan gemäss Vorlage des Palliative care NETZWERK ZH/SH [5] festgehalten.

- Bei Austritt wird der Kurzaustrittsbericht dem Hausarzt gefaxt oder gemailt, und dem Patienten werden Kurzaustrittsbericht, Überweisungsrapport der Pflege und Betreuungsplan für Hausarzt und Spitex mitgegeben.

- Der definitive Austrittsbericht wird allen involvierten Ärzten (einweisender Arzt, Heimarzt, Hausarzt, Spezialist) zugeschickt.

In der zweiten Phase von November 2008 bis November 2009 wurden Patientinnen und Patienten der medizinischen Klinik gemäss dem genannten Vorgehen konsekutiv eingeschlossen, beraten und betreut.

Alle zwei Monate wurden ausgewählte Fälle mit der Projektgruppe sowie den im entsprechenden Fall involvierten, weiteren Leistungserbringern, d.h. Hausarzt, Spitex- oder Heimmitarbeiterinnen bzw. -mitarbeitern besprochen. Ziel der Fallbesprechungen war, den Soll-Prozess laufend zu evaluieren und bei Bedarf $\mathrm{zu}$ verbessern.

\section{Resultate}

In 11 Monaten wurden 40 Patienten in das Projekt eingeschlossen, 21 Frauen, 19 Männer, Durchschnittsalter 71 Jahre (Minimum 42, Maximum 88 Jahre). 38 Personen litten an einer Krebserkrankung, 1 an fortgeschrittener COPD, 1 an Herzinsuffizienz (Abb. 1). 
Der Einschluss geschah nicht wie ursprünglich vorgesehen anlässlich des Morgenrapports, sondern erst im Verlauf der Hospitalisation, durchschnittlich 11 Tage nach Spitaleintritt (Minimum 1 Tag, Maximum 61 Tage). Die durchschnittliche Überlebenszeit nach Projekteinschluss betrug 30 Tage (Minimum 1, Maximum 127 Tage).

Bei 27 der 40 Patienten war eine Spitalentlassung möglich. 21 konnten nach Hause entlassen werden, 6 in ein Pflegeheim (Abb. 1). Von diesen 6 ist eine Person bereits aus dem Pflegeheim ins Spital eingetreten. 5 von den 6 ins Pflegeheim entlassenen Patienten starben auch im Pflegeheim. Eine Patientin wurde zur Bestrahlung einer Hüftmetastase hospitalisiert und starb während der stationären Bestrahlung.

Von den 21 nach Hause entlassenen Patientinnen und Patienten wollten 17 auch zu Hause sterben, was in 16 Fällen gelang, in einem Fall mit assistiertem Suizid. Eine Patientin musste wegen Überlastung der Angehörigen für die letzten zwei Lebenswochen noch in ein Pflegeheim eingewiesen werden. 4 von den nach Hause entlassenen Patienten wollten so lange wie möglich zu Hause bleiben, aber schliesslich im Spital sterben. Der Grund für diesen Wunsch war die Befürchtung, in der terminalen Phase kleine Kinder zu stark zu belasten oder Angehörige zu überfordern. In allen 4 Fällen konnte die terminale Hospitalisation rechtzeitig erfolgen.

Von den 27 aus dem Spital entlassenen Patienten, sind also 25 (92\%) am gewünschten Ort gestorben.

Bei 13 Patienten war zum Zeitpunkt der palliativen Beratung die Krankheit so weit fortgeschritten, dass eine Entlassung nach Hause oder ins Pflegeheim nicht mehr möglich war. 4 dieser 13 Patienten wären lieber zu Hause, bzw. im Pflegeheim gestorben. Bei drei Patienten war eine Zielklärung nicht möglich: Bei 2 dieser 3 Patienten war das Gespräch wegen Verwirrung und Somnolenz infolge Hirnmetastasen verunmöglicht. Beim dritten Patienten wollten die Töchter nicht, dass mit ihrem Vater über die schlechte Prognose und übers Sterben gesprochen wird. Sie wollten ihn glauben lassen, er werde wieder gesund.

Die anderen 6 Patienten wollten zum Zeitpunkt des Gesprächs auch im Spital sterben. Vier wurden mit dem expliziten Wunsch, im Spital zu sterben, hospitalisiert, meist wegen fehlenden sozialen Netzes zu Hause.

Von den 13 Personen, die bis zum Tod im Spital blieben, haben entsprechend $60 \%$ ihr Ziel bezüglich des Sterbeorts erreicht.

\section{Diskussion}

Ziel des Projektes war, die Spitalentlassung so zu organisieren, dass Schwer- und unheilbar Kranke ihren letzten Lebensabschnitt möglichst wunschgemäss, d.h. ohne Leiden und am bevorzugten Ort verbringen können. Das Austrittsprozedere wurde in einer interdisziplinären Arbeitsgruppe aufgrund von retrospektiven Fallanalysen definiert und anschliessend prospektiv bei hospitalisierten Patientinnen und Patienten getestet und in zweimonatlichen Fallbesprechungen auf Tauglichkeit geprüft.

\section{Patienteneinschluss in spätem Stadium}

Eine palliative Beratung war bei geschätzter Lebenserwartung unter einem Jahr vorgesehen. Tatsächlich lebten die Patienten nach Projekteinschluss noch einen Monat, im Maximum noch 4 Monate. Bei einem Drittel der Patienten war der Allgemeinzustand zum Zeitpunkt der Beratung so schlecht, dass eine Entlassung aus dem Spital nicht mehr möglich war und der Tod durchschnittlich 11 Tage später im Spital eintrat. Fast die Hälfte der nicht mehr entlassenen Patienten war in den 12 Monaten vor dem Tod schon einmal hospitalisiert und hätte bei früherer, palliativer Beratung eher am gewünschten Ort und auf gewünschte Art sterben können.

Es ist bekannt, dass medizinische Fachleute das Thema Sterben und Tod so weit wie möglich hinausschieben, um die Patienten nicht zu brüskieren. Andererseits sind sich die meisten Patienten bewusst, dass ihr Leben früher oder später zu Ende geht und auch bereit, über ihre Prioritäten und Wünsche bezüglich des Lebensendes zu sprechen. Sicher brauchen solche Ge-

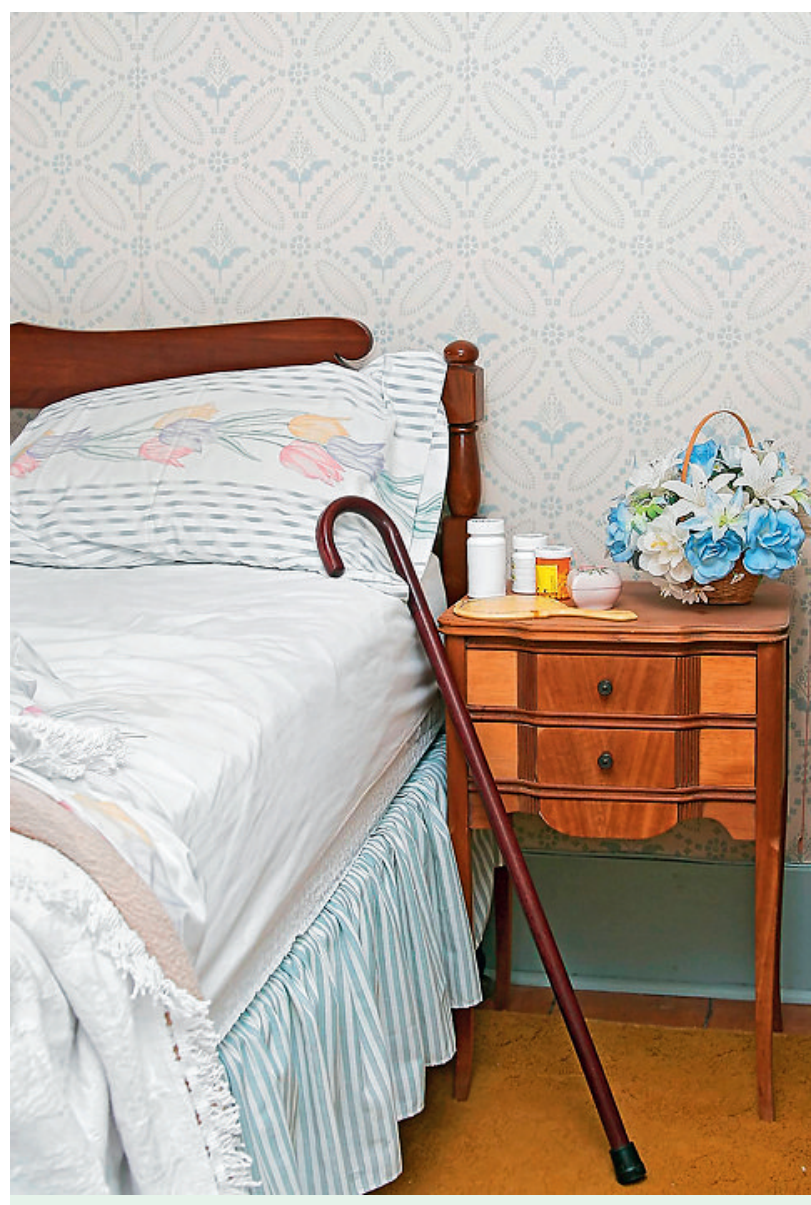

Viele Patienten wollen zu Hause sterben. Im Pilotprojekt konnte dieser Wunsch durch ein gutes Austrittsmanagement fast immer erfüllt werden. 
spräche, gerade in früheren Krankheitsstadien, Erfahrung und Fingerspitzengefühl und sollten von entsprechend ausgebildeten Fachleuten geführt werden. Würden Patientinnen und Patienten, wie ursprünglich vorgesehen, in einem früheren Stadium palliativ beraten, könnten wahrscheinlich unerwünschte terminale Hospitalisationen bei einem weit grösseren Teil der Menschen vermieden werden.

\section{Die Zusammenarbeit zwischen stationär}

\section{und ambulant tätigen Leistungserbringern hat}

\section{sich signifikant verbessert}

\section{Ziele der Patienten werden erreicht}

Trotz stark fortgeschrittenen Krankheitsstadiums gelang bei zwei Dritteln der Patienten eine Entlassung, meist nach Hause. 80\% der nach Hause entlassenen Patienten wollten auch zu Hause sterben, was mit vielen Ergebnissen von Umfragen im In- und Ausland übereinstimmt [1]. Bis auf eine Ausnahme konnte dieser Wunsch dank vorausschauender Organisation und Instruktion des Betreuungsteams inklusive proaktiver Unterstützung der Angehörigen auch erfüllt werden. Hier zeigt dieses Projekt eine ganz wesentliche Verbesserung zur sonst bekannten Statistik, die zeigt, dass weniger als ein Fünftel der Menschen zu Hause sterben kann. Wahrscheinlich konnten dank der Vorbesprechung möglicher Komplikationen, dem Notfallplan und dem Bereitstellen von Notfallmedikamenten vor Ort auch andere Ziele, wie gute Kontrolle von Schmerzen, Atemnot und andere Leiden in einem wesentlich höheren Anteil kontrolliert werden, als sonst üblich.

\section{Zusammenarbeit stationär - ambulant}

Die Fallbesprechungen, aber auch andere Rückmeldungen von Hausärzten, Spitex und Pflegenden aus Pflegeheimen zeigten, dass eine strukturierte und standardisierte Austrittsplanung mit telefonischer Kontaktaufnahme vor Austritt und Zustellung des Betreuungsplanes die Zusammenarbeit wesentlich verbessert. Einige Abläufe wurden im Verlauf des Projektes korrigiert.

In der Regel wurden die Vorschläge für die Betreuung des Patienten zu Hause oder im Pflegeheim sowie das Angebot, auch nach der Entlassung bei fehlender Erreichbarkeit oder Verfügbarkeit des Hausarztes Unterstützung zu bieten, als hilfreich betrachtet. In einigen Fällen löste das Angebot zuerst etwas Skepsis aus, die sich jedoch nach den ersten Kontakten legte und in eine konstruktive Zusammenarbeit mündete. Bei den 40 Patienten waren 31 verschiedene Hausärzte, 9 verschiedene Spitex-Organisationen und 3 Pflegeheime involviert. Bei wiederholter Zusammenarbeit konnte eine signifikante Steigerung der Effizienz der Zusammenarbeit beobachtet werden. Die Inanspruch- nahme von Unterstützung war sehr unterschiedlich und einerseits von der Betreuungskomplexität, andererseits vom Engagement der Grundversorger abhängig. In zwei Fällen erfolgte kein weiterer Kontakt mit dem Palliative care-Konsiliararzt. In den meisten Fällen kam es zu telefonischen Konsilien mit dem Hausarzt sowie telefonischen Kontakten mit den Patienten, manchmal auch zu Hausbesuchen, v. a. wenn schwer kontrollierbare Symptome auftraten oder der Hausarzt abwesend oder nicht abkömmlich war. Durchschnittlich erfolgten nach Spitalentlassung in den 42 Tagen, die die nach Hause entlassenen Patienten überlebten, noch 7 Kontakte zu Hausarzt, Spitex oder Patient.

Mit dem Ziel, die Spitex durch spezialisierte Pflegefachleute zu unterstützen, wurden Kriterien für den Beizug der Onko Plus verabschiedet. Die Pflegenden des Gesundheitszentrums Zürcher Oberland (GZO) haben bei gut der Hälfte der nach Hause entlassenen Patienten den Beizug der Onko Plus vorgeschlagen, was dann aber nur bei 5 der 21 nach Hause entlassenen Patienten geschah. Einerseits ist es für die Mitarbeitenden der Spitex unbefriedigend, die schwierigeren und vielleicht auch interessanteren Aufgaben abzugeben und sich nur noch der Grundpflege zu widmen. Andererseits steht auch die Finanzierung der Onko-Plus-Leistungen einer fruchtbaren Zusammenarbeit mit der Spitex im Weg. In den meisten Gemeinden wird aufgrund entsprechender Vereinbarungen der öffentliche, nicht über die Krankenversicherer gedeckte Teil der Leistungskosten der Onko Plus aus dem Budget der lokalen Spitex bezahlt werden. Für die $\mathrm{Zu}-$ kunft müssen die Beizugs-Kriterien aufgrund der laufenden Erfahrungen spezifiziert werden, die spezialisierten Fachleute sollten die Pflegenden der Spitex persönlich kennen und vorwiegend in der gleichen Region arbeiten und die Leistungskosten dürfen nicht direkt dem Spitexbudget belastet werden. Denkbar ist, dass die Gemeinden einer Region gemeinsam ein spezialisiertes Team mit einer Pauschale pro Einwohner finanzieren oder dass der Kanton den öffentlichen Anteil spezialisierter Pflegeleistungen deckt.

\section{Auswirkung auf Kosten}

Die vorausschauende Planung und Organisation der Betreuung von Schwerkranken führt nicht nur in diesem Projekt, sondern auch in verschiedenen, prospektiv randomisierten Studien zur Vermeidung von Rehospitalisationen, Notfallbehandlungen und damit je nach Studie zu Kosteneinsparungen bis 50\% im letzten Lebensjahr [6]. Die zusätzlichen Kosten von ca. 600 Franken für die Entlassungsplanung werden meist schon durch die Vermeidung einer einzigen Notfallsituation kompensiert.

Auch aus Sicht des Spitals ist die palliative Beratung wahrscheinlich mindestens kostenneutral. Nach Klärung der Ziele und Fokussierung auf eine palliative Betreuung kann in etlichen Fällen von weiteren technischen Leistungen abgesehen werden und der Austritt wird, falls noch möglich, beschleunigt. Unter 
DRG-Finanzierung ist die Vermeidung von Rehospitalisationen mindestens kurz nach Spitalaustritt auch im Interesse des Spitals, weil in diesem Fall keine zusätzliche Vergütung mehr vorgesehen ist.

\section{Konklusion}

Das vorliegende Projekt zeigt, dass eine gute Planung und Organisation der Spitalentlassung von schwerkranken Patientinnen und Patienten in palliativer

\section{Danksagung}

Der Gesundheitsdirektion des Kantons Zürich danken wir für die Initiierung und Mitfinanzierung dieses Projektes. Prof. Dr. Johann Steurer vom Horten Zentrum für praxisorientierte Forschung und Wissenstransfer gab uns wertvolle Informationen bzgl. Methodik und weiterführender Studien. Dr. Andreas Gattiker und die ganze Spitalleitung des GZO haben das Projekt von Anfang an mit grossem Engagement unterstützt.

\section{Eine gute Planung der Spitalentlassung ermöglicht es schwerkranken Patienten, ihr Leben am gewünschten Ort zu beenden}

Situation in den meisten Fällen ermöglicht, dass diese Patienten ihr Leben am gewünschten Ort beenden können und sich die Zusammenarbeit zwischen stationär und ambulant tätigen Leistungserbringern signifikant verbessert. Damit nicht nur ein kleiner Teil dieser Patientengruppe von einer guten Austrittsplanung profitiert, müssen innerhalb des Spitals personelle Ressourcen für diese Aufgaben sowohl bei der Pflege als auch bei den Ärzten und beim Sozialdienst geschaffen werden. Die entsprechenden Fachleute müssen eine Ausbildung in Palliative care durchlaufen und den ambulanten Bereich gut kennen. Denkbar ist, dass die Fachleute aus dem Spital die ambulanten Leistungserbringer bei schwieriger Symptomkontrolle auch nach Entlassung der Patienten konsiliarisch unterstützen. Zudem können sie spitalintern bei der palliativen Betreuung jener Patienten beigezogen werden, die nicht mehr entlassen werden können (interner, palliativer Konsiliardienst).

Auf diese Weise kann ein Spital die palliative Betreuung im ganzen Einzugsgebiet verbessern und durch eine engere Zusammenarbeit mit Hausärzten und Spitex auch den Prozess der Spitaleinweisung optimieren oder Hospitalisationen vermeiden.

\section{Literatur}

1 Clark J. Freedom from unpleasant symptoms is essential for a good death. BMJ. 2003;327:180.

2 Deandrea S, Montanari M, Moja L and Apolone G. Prevalence of undertreatment in cancer pain. A review of published literature. Ann Oncol. 2008;19:1985-91.

3 Van den Block L, Deschepper R, Drieskens K, Bauwens S, Bilsen J, Bossuyt $\mathrm{N}$ and Deliens L. Hospitalisations at the end of life: using a sentinel surveillance network to study hospital use and associated patient, disease and healthcare factors. BMC Health Services Research. 2007;7:69.

4 Unterstützung und Koordination in der ambulanten Palliative Care. Palliative care Netzwerk Zürich; 2007. www.pallnetz.ch/de/koordination.php

5 www.pallnetz.ch/de/dokumente.php

6 Brumley R, Enguidanos S, Jamison P, Seitz BW, Morgenstern N, Saito S et al. Increased Satisfaction with Care and Lower Costs: Results of a Randomized Trial of In-Home Palliative Care. J Am Geriatr Soc. 2007;55:993-1000. 